\title{
Publisher Correction: Epigenetic modulation of a hardwired 3D chromatin landscape in two naive states of pluripotency
}

Yaser Atlasi, Wout Megchelenbrink, Tianran Peng, Ehsan Habibi, Onkar Joshi, Shuang-Yin Wang, Cheng Wang,

Colin Logie (D), Ina Poser, Hendrik Marks (D) and Hendrik G. Stunnenberg (D)

Correction to: https://doi.org/10.1038/s41556-019-0310-9, published online 29 April 2019

In the version of the article originally published, extra lines were displayed in Fig. 7. Fig. 7a contained a solid black line that extended into panel b, and Fig. 7c contained two extra scale bars on the left. These have been removed from the figure. The errors have been corrected in the HTML and PDF versions of the article.

Original

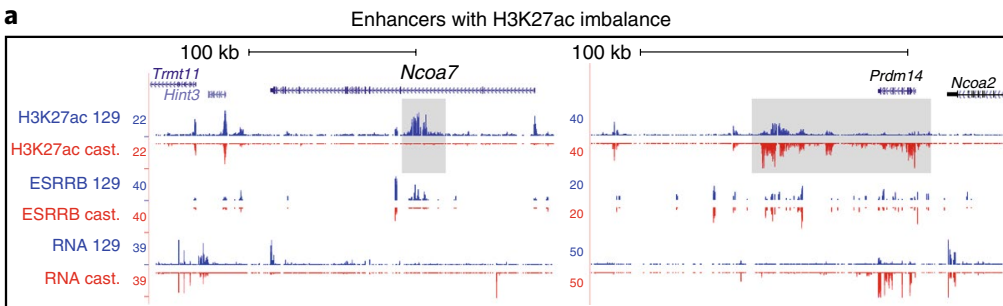

Corrected

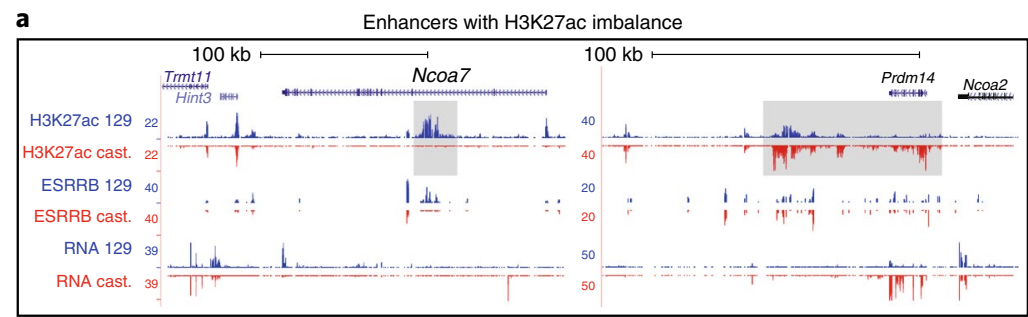

Fig. 7a | Orginal and Corrected. 


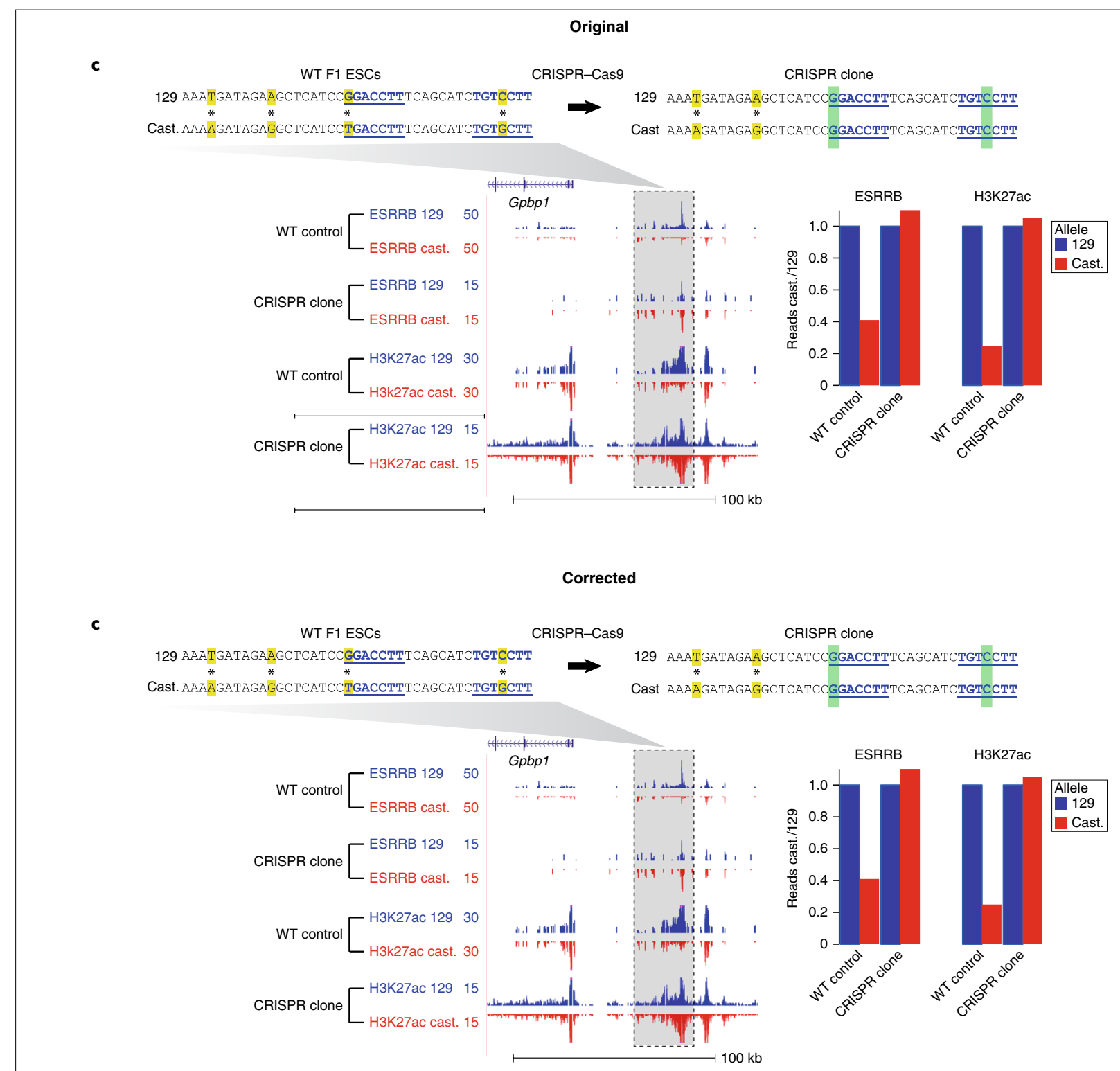

Fig. 7c| Orginal and Corrected.

Published online: 16 May 2019

https://doi.org/10.1038/s41556-019-0341-2 